\title{
Il mistero della galaverna
}

\author{
Matilde Paoli
}

PUBBLICATO: 26 MARZO 2012

\section{Quesito:}

Manuela Bonfatti dalla provincia di Reggio Emilia e Giorgio Giordano da Torino ci chiedono quale sia l'etimologia di galaverna; più in particolare Fabrizio Conti dalla provincia di Firenze ci domanda se è possibile ipotizzare un imparentamento etimologico tra il termine e la forma francese verglas. Infine Marco Orlandi da Lodi si interroga se galaverna sia da considerare un termine regionale/dialettale o se invece abbia "dignità di Italiano ufficiale". Segnaliamo che una risposta di Luca Serianni sul significato di galaverna era già apparsa sul n.I9 (ottobre 1999) della Crusca per voi.

\section{Il mistero della galaverna}

Quando lo scorso mese, nel fitto dell'inverno, io venni da te, carissimo Oreste, vidi, girando l'occhio lungo tutta la strada e la sfogata campagna, lo spettacolo pittoresco della galaverna; dove a creste o zighizaghi, dove a nastri o trine, quando a pizzi o frange, e quando a pendagli o rotoletti, come fiori d'avellani o di noci, secondo l'intrecciata e varia conformazione o configurazione de' rami negli alberi e nelle siepi...

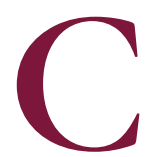

o si Prospero Viani in Calaverna o galaverna, una delle sue Lettere filologiche e critiche indirizzata al conte Oreste Biancoli e datata febbraio i 874.

Possiamo trovare una descrizione meno impressionistica di cosa si intenda in italiano per galaverna nel Breve dizionario di termini meteorologici in cinque lingue, edito nel 1963 a cura del Ministero difesa-aeronautica, dove al lemma galaverna si rimanda a ghiaccio granuloso che è così definito:

Formazione di ghiaccio, detta anche galaverna, costituita da granuli, più o meno separati da inclusioni di aria, e talvolta con ramificazioni cristalline; friabile e bianca. Si produce per congelamento rapido di piccolissime goccioline sopraffuse di nebbia o di nube: al suolo, sugli oggetti esposti al vento, e particolarmente dal lato sopravvento; in quota, sugli aeromobili e particolarmente sulle superficie esposte al vento relativo.

Nello stesso dizionario sono elencate anche altre voci che si trovano in stretta correlazione con galaverna e l'equivalente ghiaccio granuloso, ovvero vetrone, con rimando a ghiaccio vitreo, e soprattutto calabrosa - che come vedremo è fortemente connessa con galaverna - con rimando a ghiaccio compatto, cosi definito:

Formazione di ghiaccio, detta anche calabrosa, costituita da strati amorfi compatti, semitrasparenti e molto tenaci. Si produce per congelamento rapido di goccioline sopraffuse, relativamente grosse, di nebbia o di nube: al suolo, sugli oggetti esposti al vento, dove può formare depositi di grande spessore; in quota, sugli aeromobili e particolarmente sulle superficie esposte al vento relativo.

Delle voci vengono riportati gli equivalenti in francese, inglese, spagnolo e tedesco.

Credo sia evidente il tentativo di sostituire termini (galaverna e calabrosa), riconducibili come vedremo al lessico tradizionale di alcune aree, con altrettanti sintagmi di lingua la cui testa, ghiaccio, è 
variamente modificata sul modello del francese (givre + mou/granuleux, + transparent, + dur/compact) o dello spagnolo (cenceñada + blanda, + transparente, + dura) o anche, parzialmente, dell'inglese (soft rime, hard rime) e del tedesco (Rauhreif, Rauhfrost), rispondendo all'esigenza di simmetria tipica della tassonomia scientifica.

Che si trattasse di voci appartenenti a varietà locali è testimoniato non solo dai dizionari dialettali ottocenteschi, ma anche da opere coeve o di qualche decennio precedenti al dizionario, come l'AIS pubblicato dal 1928 al I940, e in particolare nella carta 375, che indaga i nomi della brina (Reif in tedesco), e nella carta 376 il cui titolo 'galaverna' (sia Rauhreif che Rauhfrost) è indicato tra apici per segnalarne l'incerta attribuzione all'italiano. Riportiamo la definizione dell'area di diffusione di galaverna, calaverna (con le varianti galiverna e calinverna) - ma anche di calabrosa ebrosa - che ricaviamo dallo studio di Gabriella Giacomelli, Calaverna e brosa nei dialetti italiani (I979), basato sulle testimonianze dell'AIS e dei repertori dialettali.

[il tipo calaverna] ha un'ampia estensione nella parte occidentale dell'Italia settentrionale e in una zona del Veneto meridionale strettamente associata all'area emiliano-romagnola e all'area centrale [Marche, Umbria e Toscana appenninica]: in particolare si nota che il tipo con [g] iniziale è piemontese, ligure, emiliano-romagnolo, quello con $[\mathrm{k}]$ iniziale veneto e italiano centrale. (p. I2I)

[il tipo calabrosa è testimoniato] nell'Emilia occidentale, come nella Lombardia orientale e nel Trentino... (p.rzo)

L'ingresso in italiano, o meglio nella terminologia scientifica, di galaverna e calabrosa, termini di tradizione non toscana, caratterizzati peraltro dalla particolare dissimmetria della consonante iniziale, si deve al fisico Ciro Chistoni, il quale intitolò Brina, galaverna e calabrosa una nota del i 8 giugno I9ıo indirizzata alla Reale Accademia delle Scienze di Napoli. Chistoni, per indicare i due fenomeni diversi di congelamento della nebbia, aveva seguito l'esempio del meteorologo prussiano Richard Assmann che, durante un'ascesa sul Monte Brocken (I4Io m.) in Prussia, aveva scoperto che il fenomeno di congelamento di gocce d'acqua sopraffuse in quota poteva manifestarsi anche sotto forma di ghiaccio omogeneo. Egli, non potendo usare, per designare l'idrometeora, il termine Rauhreif, che in Prussia indicava il fenomeno già noto in forma di ghiaccio granuloso, lo denominò Rauhfrost, sinonimo in uso nell'area austriaca. Chistoni, cremonese, usò lo stesso procedimento stabilendo in italiano le equivalenze, galaverna (presente nel suo dialetto) = Rauhreif e calabrosa = Raufrost.

In realtà la forma maschile galaverno era già presente nella lessicografia di lingua, la troviamo nel secondo volume del Tommaseo-Bellini ( I869) come "il ghiaccio sugli alberi voce usit. a Boscolungo [Appennino pistoiese]e al Monte Amiata [...] in Piemonte Galaverna...". Ecalaverno "i diaccioli che si vedono pendenti dagli alberi e da' tetti" come voce della montagna pistoiese compare anche nella terza edizione postuma del Vocabolario della lingua italiana di Pietro Fanfani (I89I). La presenza inaspettata nello stesso vocabolario della forma femminile con $g$ - iniziale, definita come "acquerugiola, rugiada o nebbia che divien quasi solida per il gelo improvviso che la coglie sui rami degli alberi", è dovuta all'introduzione, dichiarata nell'avvertenza all'edizione, di voci tratte da opere successive all'edizione precedente (I865) e in particolare da repertori tecnici tra cui il Vocabolario di agricoltura di Eugenio Canevazzi, dove troviamo, con definizione pressoché identica, il lemma galaverna. Petrocchi nel Nóvo dizionario universale della lingua italiana (I89I) riporta solo galaverno e calaverno nella parte "fuori dall'uso", siglate rispettivamente $(\mathrm{T}=$ Tommaseo $)$ e $(\mathrm{F}=$ Fanfani) a dichiararne la provenienza, e tace invece della forma femminile. La ripropone il marchigiano Alfredo Panzini nel ig05, anno della prima edizione del suo Dizionario moderno, supplemento ai dizionari italiani: "galaverna cosi chiamano con voce dialettale in Romagna il nevischio gelato e minuto, quello che i francesi dicono verglas: e significa 
altresi la brina". Resistenza alla forma con l'iniziale sonora troviamo nello Zingarelli che, dalla prima edizione (I9I7) e fino alla nona (I965) ha il lemma calaverna con il valore, glossato come toscano, di 'ghiacciuolo sui rami degli alberi', già della forma maschile in Fanfani e Tommaseo, e l'altro, glossato come emiliano, di 'nebbione'; troviamo anche galaverno, come in Tommaseo, definito dialettale.

Solo a partire dalla decima edizione (I970) Zingarelli registra il lemma galaverna o calaverna e il significato "rivestimento di ghiaccio su oggetti al suolo esposti al vento, costituito da granuli provenienti da rapido congelamento di piccolissime goccioline d'acqua sopraffuse"; contemporaneamente compare per la prima volta calabrosa, senza alcuna annotazione rispetto all'uso o alla varietà, come "rivestimento di ghiaccio tenace, compatto, traslucido, movente dal rapido congelamento di goccioline d'acqua sopraffuse" (in entrambe le definizioni si noteranno analogie col Breve dizionario di termini meteorologici già citato); scompaiono invece le forme maschili. Più o meno contemporanea l'attestazione nella prima edizione del Devoto-Oli (I97I) dove calaverna (o galaverna) vale "Brina o nebbia che cristallizza sui rami e sulle foglie formando dei lunghi aghi; nell'Italia settentrionale sinonimo di brina, brinata, nevischio"; anche questo dizionario registra calabrosa.

Da allora i due lemmi compaiono in gran parte dei dizionari con analoghe definizioni; varia invece la valutazione della voce: nello Zingarelli dal 200 calaverna, lemma principale dal ı998, e galaverna, sono glossati come appartenenti al lessico tecnico-scientifico della meteorologia, mentre calabrosa approda all'edizione 2012 senza annotazioni; anche nel De Felice-Duro, che non ha calabrosa, calaverna/galaverna pertengono al lessico della meteorologia; in Palazzi-Folena è un regionalismo, mentre calabrosa è privo di annotazioni; in GRADIT e nel Vocabolario Treccani è calabrosa a essere valutato come un regionalismo di area settentrionale, mentre calaverna/galaverna non hanno marche; infine dal DISC 1997 al Sabatini Coletti 2008 sia calaverna/galaverna sia calabrosa sono glossati come regionalismi.

Nella tradizione letteraria, stando ai corpora esaminati, calabrosa è assente; per calaverna l'unica attestazione sembra essere quella, riportata in GDLI, di Fabio Tombari (marchigiano!) in Cronache di Frusaglia (1928). Più numerose le attestazioni digalaverna, la prima delle quali è attribuita al modenese Ludovico Antonio Muratori nelle sue Dissertazioni sopra le antichitá italiane (I75I-55), vol. III-8o ("I Modenesi non solamente al pari degli altri Italiani chiamano 'brina' il latino 'pruina', ma anche 'galaverna' essa brina gagliarda congelata ne gli alberi."). GDLI riporta inoltre le testimonianze del vercellese (di Saluggia) Giovanni Faldella - che in Madonna di fuoco e Madonna di neve (I888) ha in realtà la forma galiverna -, dell'emiliano Corrado Govoni in L'inaugurazione della primavera (1915) e del cuneese Cesare Pavese in La luna e i faló (1950). La consultazione del corpus LIZ aggiunge una testimonianza per il XVI secolo nel resoconto della Navigazione di Sebastiano Cabota [sic], dalla raccolta Navigazioni e viaggi di Giovan Battista Ramusio, che narra una spedizione nelle terre russe progettata da Caboto per conto della corona inglese, e quella più tarda di Alfredo Oriani, faentino, in Quartetto (i 883 ). Non sarà sfuggita l'appartenenza degli autori all'area geografica in cui la forma risulta tradizionale.

E l'uso attuale? Innanzi tutto si conferma, rispetto alla variante "toscana" calaverna, il successo di galaverna che si mostra piuttosto vitale nell'ambito della meteorologia, e compare, spesso affiancato dal sintagma ghiaccio granuloso, in dizionari e contributi di carattere divulgativo reperibili in rete. Non ha prodotto risultati invece la ricerca di galaverna (e calabrosa) nel sito del Servizio meteorologico dell'aeronautica militare; né lo troviamo nel Corso di meteorologia aeronautica dell'Ente Nazionale di Assistenza al Volo laddove, trattando il fenomeno dell'icing o "contaminazione da ghiaccio", vengono descritti i principali tipi di ghiaccio, nelle cui denominazioni (ghiaccio brinoso, ghiaccio granuloso, 
ghiaccio vetrone) è possibile ravvisare la tipologia tripartita del Breve dizionario del I963; nemmeno appare nell'analoga classificazione dei tipi di ghiaccio nel sito di meteorologia collegato alla facoltà di Ingegneria della Protezione Civile dell'Università di Enna "Kore": ghiaccio brinoso, ghiaccio vetroso o vetrone, ghiaccio misto.

Anche i quotidiani a diffusione nazionale sono utili sia per affermare la scarsa vitalità di calabrosa (mai attestata) sia per testimoniare l'affermazione di galaverna (mai calaverna). Delle testimonianze presenti fino dai primi anni negli archivi del "Corriere" e di "Repubblica" riporto solo la più lontana negli anni, tratta da un articolo di Enzo Biagi sulla strage di San Benedetto Val di Sambro:

Conosco quei posti. Ci sono stato durante la guerra. Ricordo il capostazione di Ca' di Landino, che costruiva treni in miniatura, il velluto rosso nei sedili delle prime classi, e la locomotiva fischiava cupa imboccando la galleria. So com’è il paesaggio di questi giorni: la galaverna imbianca gli alberi, l'aria tersa odora di legna bruciata, nella notte di Natale si usa accendere i falò: e c'è quasi sempre la luna sui monti. Sono paesi dell'Appennino emiliano, e quella che si ritrova in piazza o all'osteria è la mia gente: di là dal crinale, la Toscana. (Quell'ora fatale sul treno del sud "Repubblica" 25 dicembre 1984)

Galaverna quindi è un termine che ha "dignità di lingua", per citare il nostro utente, e in particolare pertiene al settore della meteorologia, ma nello stesso tempo, come credo si possa desumere anche dal passo di Biagi, mantiene una dimensione regionale, un forte legame con la realtà in cui è parola della tradizione.

Veniamo ora all'etimologia di calaverna/galaverna. Lindagine sull'origine di questa forma si presenta molto complessa e sono state avanzate numerose proposte da parte degli studiosi. Prospero Viani, nella lettera citata in apertura, fa un elenco semiserio dei diversi tentativi succedutisi a partire dal XVII secolo fino ai suoi giorni, eleggendo come ipotesi più probabile l'interpretazione della voce come un composto latino caligo-hiberna.

Nel 1956, quando Raffaele Giacomelli riprese la questione in Per l'etimologia di galaverna e di calabrosa, poteva aggiungere altra materia all'elenco del Viani, ma doveva anche riferire come, solo pochi anni prima (195I), Angelico Prati nel Vocabolario etimologico della lingua italiana ritenesse che non si fosse ancora trovata una spiegazione soddisfacente. Dal canto suo Giacomelli ripropose la connessione con i derivati del latino caligo caliginis, presenti nei dialetti veneti a indicare una sorta di nebbia, sia per galaverna/calaverna che per calabrosa; per il secondo elemento rimandava a hibernus e abrosa (e varianti) che nell'area nordorientale vale(va) 'brina'.

Anche Gabriella Giacomelli, più di venti anni dopo, nel contributo citato che richiama volutamente nel titolo lo studio del suo omonimo, giunge a risultati importanti per lo studio etimologico: il tipo primario è da ritenersi calaverna, come è da considerarsi originaria la $a$ in seconda sillaba, mentre le forme con la $i$ sono secondarie; forma secondaria sarebbe anche calabrosa, frutto di incrocio tra calaverna e brosa. L'autrice ripropone, alla luce delle sue conclusioni, una sintesi della questione dell'etimologia di calaverna e calabrosa, individuando tre direttrici: la latina, la gallica e la mediterranea. La direttrice latina considera la voce un composto caligo-hiberna ed è, come abbiamo visto, considerata la più attendibile da Viani e da Giacomelli; a parere della studiosa però è resa poco persuasiva sia dai risultati della sua analisi, che affermano essere primaria la presenza della $a$ in seconda sillaba, sia per la rarità dei composti del genere in latino e neoloatino.

Queste difficoltà sono superate nella proposta "gallica" di Vittorio Bertoldi che, in Problemi d'etimologia, (1936) analizza la forma come cal-av-erna avvicinando l'elemento cal-all'antico irlandese caile 'macchia' risalente alla stessa radice indoeuropea del latino calidus. La teoria mediterranea infine è suggerita dal DEI che rimanda a un tema *calabro-/*galabro- 'concrezione calcarea o ghiacciata' da cui calabrosa, rispetto al quale calaverna sarebbe una formazione aggettivale. Anche questa teoria è messa 
in crisi dalla stessa Giacomelli che considera calabrosa frutto di incrocio tra brosa e calaverna.

Lo studio di Gabriella Giacomelli è coevo alla pubblicazione del DELI, il quale per galaverna aggiunge alla lista delle proposte etimologiche l'ipotesi di J.U. Hubschmied - che vedeva una connessione con la radice indoeuropea *gel- attraverso una forma gallica *gala gemerina, da intendersi come 'gelo invernale' ("Vox Romanica" III, I938, I3I-I33) - e quella di Giovanni Alessio, che risaliva all'alternanza mediterranea *calabo-/*galabo- con suffisso - erna frequente in voci di sostrato ("Revue de linguistique romaine" XVII, I952, 62).

Nel LEI viene accolta la proposta di Schuchardt che vedeva in calaverna la radice indoeuropea *cal(dal doppio significato di 'freddo' e 'caldo') con infisso - av-di origine celtica, attestato nelle Alpi, per esempio con i nomi di luogo, più il suffisso -erna, che si applica soprattutto ai fenomeni naturali. Più recentemente, ne L'etimologico, Alberto Nocentini pensa per calaverna, galaverna a una "formazione romanza di origine latina dal latino volgare *calaberna $(\mathrm{m})$ derivato dalla radice cal- di calorem e caligo" la cui radice sarebbe stata modificata "da un suffisso -ber- seguito dai suffissi aggettivali -num e -osum da cui calabrosa", tornando quindi a considerare calabrosa tipo primario.

L'unica cosa che sembra certa a questo punto è che per galaverna/calaverna si possa escludere un rapporto di derivazione con il francese verglas suggerito dal nostro utente. È vero però che verglas, composto di verre 'vetro' e glas, altra forma di glace 'ghiaccio', come già ricordava Raffaele Giacomelli, ha un equivalente nel dialetto parmense vedergiás 'vetroghiaccio' che è a sua volta connesso con il vetriore fiorentino e il solvetro o sovvetro mugellano che indicano il vetrone o ghiaccio vetroso.

Secondo il nostro parere, la ricerca etimologica ci lascia a tutt'oggi senza una risposta certa; possiamo ancora concordare con Prospero Viani che, nella lettera più volte citata, scriveva: "tu sai che queste cose, come l'etimologie, mentre crediamo d'averle in pugno ne sguisciano via come l'anguille".

Chi vuole, può, sapendo che non è scienza, vedere in galaverna una "gala invernale", come Ciro Chistoni il quale scrisse: "La Galaverna è quasi definita dallo stesso nome: è una specie di addobbo invernale di tutti gli oggetti esposti al libero cielo."

Nota bibliografica:

- AIS K. Jaberg - J. Jud, Sprach- und Sachatlas Italiens und der Südschweiz, Zofingen, Ringier \& Co., I928-I940

- Bertoldi Vittorio, Problemi d'etimologia, "Zeitschrift für romanische Philologie", LVI (r936), pp. I 84 -I 88

- Bilancini Raul, Breve dizionario di termini meteorologici in cinque lingue, Roma, Ministero difesaaeronautica, Ispettorato telecomunicazioni e assistenza al volo, I963

- Giacomelli Gabriella, Calaverna e brosa nei dialetti italiani, "Archivio per l'Alto Adige" LXXIII, I979, pp. II7-I32

- Giacomelli Raffaele, Per l'etimologia di galaverna e di calabrosa, "Lingua nostra", XVII, fasc. 2, giugno 1956 , pp. 38-42

\section{Cita come:}

Matilde Paoli, // mistero della galaverna , "Italiano digitale", 2012, XI, 2019/4 (ottobre-dicembre)

DOI: $10.35948 / 2532-9006 / 2020.3263$ 